\title{
Effects of precuing horizontal and vertical dimensions on right-left prevalence
}

\author{
ROBERT W. PROCTOR \\ Purdue University, West Lafayette, Indiana \\ IRING KOCH \\ Max Planck Institute for Human Cognitive and Brain Sciences, Munich, Germany \\ and \\ KIM-PHUONG L. VU \\ California State University, Long Beach, California
}

\begin{abstract}
When stimuli and responses can be coded along horizontal and vertical dimensions simultaneously, a right-left prevalence effect is often obtained for which the advantage for a compatible mapping is larger on the horizontal dimension than on the vertical dimension. The present study investigated the role of preparatory processes in this right-left prevalence effect using a method in which the relevant dimension was cued at short and long intervals prior to presentation of the target stimulus. In three experiments, the right-left prevalence effect did not vary significantly in magnitude as a function of cue-target interval, suggesting that the effect is due primarily to relative salience of the horizontal and vertical codes, as determined by the task structure, and not to a greater ease of attending to the horizontal dimension.
\end{abstract}

When stimulus and response locations are arrayed at the ends of one of the major diagonals of imaginary squares, they can be coded along both the horizontal and vertical dimensions. Subjects can be instructed to respond on the basis of the stimulus' horizontal or vertical location. When the responses and the stimuli are placed along the same diagonal, the mapping of stimuli to responses can be either compatible or incompatible on both dimensions. However, when the responses and the stimuli are placed along opposite diagonals, the mapping can be compatible on either the horizontal or the vertical dimension, but not both. A common finding is that even if subjects are instructed in terms of the vertical dimension, performance is better when horizontal compatibility is maintained than when vertical compatibility is maintained, a phenomenon that Nicoletti and Umiltà (1984) named the right-left prevalence effect.

This research was initiated while R.W.P. was a visiting scientist at the Max Planck Institute for Psychological Research, Munich. We thank Wolfgang Prinz for his support and encouragement of this collaborative research. We also thank Erik Altmann, Bernhard Hommel, and an anonymous reviewer for their comments on an earlier version of this manuscript. I.K. is now at the Institute of Psychology, RWTH Aachen. Correspondence regarding this manuscript should be addressed to R. W. Proctor, Department of Psychological Sciences, Purdue University, 703 Third Street, West Lafayette, IN 47907 (e-mail: proctor@psych.purdue .edu).

Note-This article was accepted by the previous editorial team, when Colin M. MacLeod was Editor.
Accounts of the right-left prevalence effect tend to concentrate on automatic or strategic processes. Nicoletti and Umiltà (1984, 1985; Nicoletti, Umiltà, Tressoldi, \& Marzi, 1988) focused on the former because they obtained an advantage for responses compatible on the horizontal dimension compared with those compatible on the vertical dimension, even though their subjects were instructed in terms of the vertical dimension. Nicoletti and Umiltà $(1984,1985)$ proposed that the right-left prevalence effect was due to an inherent advantage for horizontal coding over vertical coding, and Nicoletti et al. (1988) and Umiltà and Nicoletti (1990) suggested even more strongly that horizontal codes prevent vertical codes from being formed. In contrast, Hommel (1996) emphasized primarily strategic processes, arguing that the right-left prevalence effect in Nicoletti and Umiltà's studies was due to their subjects' not heeding the vertical instructions and choosing instead to attend to the horizontal dimension. Hommel (1996) suggested that this strategy of attending to the horizontal dimension was adopted because the horizontal codes may have been available sooner than the vertical codes in Nicoletti and Umiltà's experiments (see also Rubichi, Nicoletti, Pelosi, \& Umiltà, 2004). As evidence for this account, Hommel showed that when subjects were urged to respond in terms of only the vertical dimension or only the horizontal dimension, an advantage for the instructed dimension was obtained, even when that dimension was vertical.

Hommel (1996) noted that although the instructions to respond on the basis of the vertical or the horizontal dimension altered the relative speed at which the two dimen- 
sions were processed (i.e., showed an advantage for the instructed dimension), there was still a right-left prevalence effect: The benefit of vertical compatibility was larger than that of horizontal compatibility under vertical instructions, and vice versa under horizontal instructions, but the former benefit was smaller than the latter. Vu and Proctor $(2001,2002)$ called this the "weak" form of right-left prevalence because it is evident only when collapsed across instructions. The asymmetry in the instruction effects indicates that even though attending to the vertical dimension can produce a benefit for that dimension, it does not eliminate the tendency for the horizontal spatial codes to be more salient than the vertical codes. Since Hommel found this weak right-left prevalence effect to be absent for unimanual responses (see Vu \& Proctor, 2001), he attributed the relative salience of the horizontal codes to a "dimensional bias" induced by using left-right effectors.

$\mathrm{Vu}$ and Proctor $(2001,2002)$ showed that a "strong" version of the right-left prevalence effect, for which the horizontal compatibility effect is larger than the vertical compatibility effect even with vertical instructions, occurs when the hands are held apart on a hand-grip apparatus, highlighting the left-right distinction. Conversely, they also demonstrated that a top-bottom prevalence effect can be produced by highlighting the vertical dimension, either through placing one hand on top of the other or by using top and bottom effectors (the ipsilateral hand and foot). Vu and Proctor (2002) established that manipulations of relative proximity of the stimulus locations along the vertical and horizontal axes have similar effects on performance, with prevalence effects being largest when the same dimension is salient for both the stimulus and response sets. According to $\mathrm{Vu}$ and Proctor (2002), the task structure determines the relative salience of the vertical and horizontal codes. Instructions to attend to a particular dimension modulate the effects attributable to relative salience by directing attention to the instructed dimension. However, although subjects can direct their attention equally well to the horizontal and vertical dimensions, this attentional process must operate on the codes determined by the task structure.

In contrast, a grouping model devised by Adam, Hommel, and Umiltà (2003) to explain results from response precuing tasks assumes that attentional processes can overcome the more automatically determined organization provided by the salient environmental features. In such tasks, four horizontal stimulus locations are mapped compatibly to four horizontal response locations. Similar to the rightleft prevalence effect, precuing the two left or two right locations yields better performance than does precuing other pairs of locations (Reeve \& Proctor, 1984). To account for this right-left precuing advantage, Adam et al.'s model assumes that grouping operations organize stimulus locations in an input buffer and response locations in an output buffer as the basic units of response selection. The model "assigns a critical role to the mode of selection by distinguishing between fast, automatic subgroup selection, and slow, effortful subgroup creation" (Adam et al., p. 315). With regard to situations producing a right-left prevalence effect, selection of the right-left sub- group would be fast and automatic, whereas that of the top-bottom subgroup would be slow and effortful.

As described, the right-left prevalence effect is defined by between-block comparisons of conditions that maintain compatibility on the horizontal and/or vertical dimension (Nicoletti \& Umiltà (1984). With this methodology, a single mapping of the two stimuli to the two responses is in effect for an entire trial block, allowing subjects to be prepared to select responses consistent with the assigned mapping on a particular dimension. This procedure thus cannot answer the question of whether the state of preparation to respond based on one dimension or the other influences the right-left prevalence effect. Consequently, the present study used a method in which the to-be-attended dimension was cued prior to onset of the target stimulus (see Figure 1), instead of being held constant within a trial block. This cuing method allows manipulation of the preparation for responding to a particular dimension by varying the time between cue onset and target stimulus onset. With a long cue-target interval, subjects can prepare to respond on the basis of the cued dimension, whereas with a short cue-target interval, there is insufficient time for full preparation. A variant of this cuing method has been successfully applied by Meiran (1996; Meiran, Chorev, \& Sapir, 2000) to examine preparatory effects in task switching. The focus in those studies was not on the right-left prevalence effect but on whether the task (vertical or horizontal) repeated or switched from the preceding trial. In contrast, our primary concern in the present study was with whether cue-based preparation would influence the right-left prevalence effect.

According to Vu and Proctor's $(2001,2002)$ relative salience account, the size of the right-left prevalence effect should not depend on the state of preparedness to respond in terms of the cued dimension. This is because the prevalence effect is a result of constraints imposed on coding by the task structure. Although performance should improve when there is time to develop an appropriate set for the cued dimension, this preparation effect should not alter the prevalence effect. In contrast, Adam et al.'s (2003) grouping model predicts that the prevalence effect should be large at short cue-target intervals and decrease at long intervals, as the slow, effortful subgroup process allows preparation for responding based on the vertical dimension.

In the present experiments, responses were made with the index fingers of the left and right hands placed on the numeric pad of a keyboard, which typically yields a rightleft prevalence effect when collapsed across horizontal and vertical instructions. The right-left prevalence effect will be defined in this case as an advantage in performance (shorter response times and fewer errors) when the horizontal dimension is cued relative to when the vertical dimension is cued. We chose to define right-left prevalence in this manner, rather than as a function of the compatibility effect for the cued dimension (i.e., a larger horizontal compatibility effect when the horizontal dimension is cued than vertical compatibility effect when the vertical dimension is cued), because with this cuing method, the instructed or cued dimension varies within a block of trials 


\section{Compatible Mapping}

Cue: Horizontal

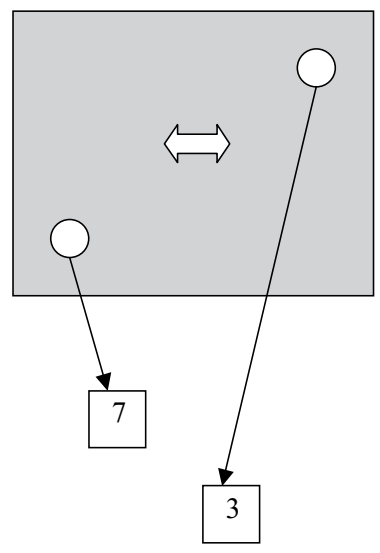

Cue: Vertical

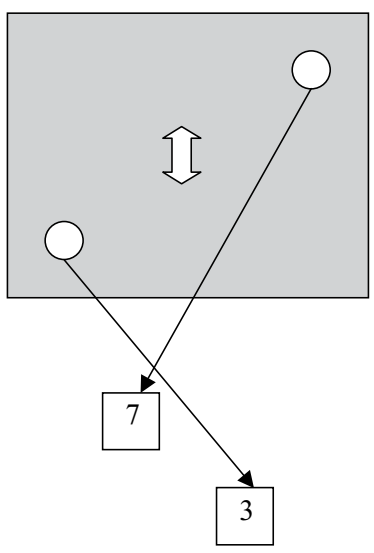

Cue: Vertical
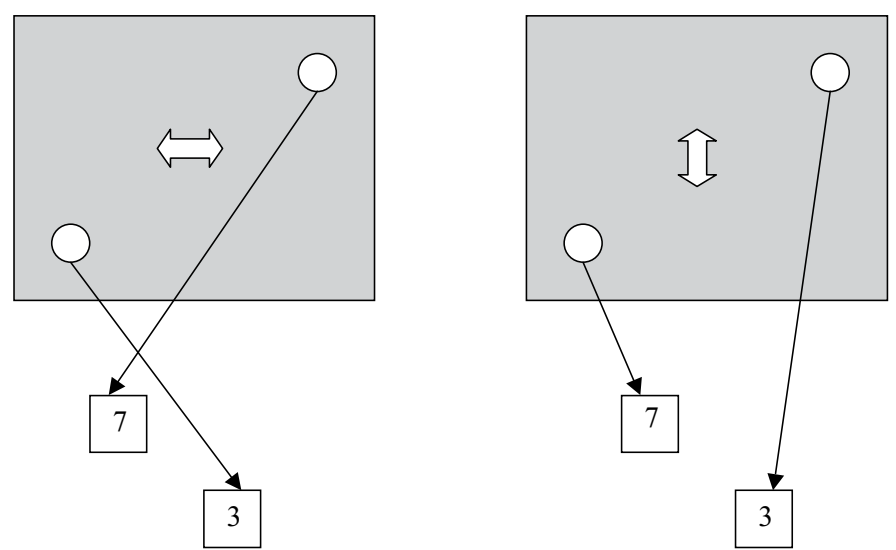

Figure 1. Examples of the compatible and incompatible mapping conditions for the horizontal and vertical cued dimensions. Half of the subjects in each mapping condition performed with the illustrated stimulus and response positions, and half with stimuli and responses arrayed along the opposite diagonals.

and the mapping of stimuli to responses is held constant for each subject. This is in contrast to previous studies of right-left prevalence in which the instructed dimension was held constant for each subject and the mapping of stimuli to responses varied.

The cuing method used in Experiments 1 and 2 examines conditions in which spatial compatibility is maintained for either the horizontal or the vertical dimension, but not both, or, in other words, ones for which the mapping rules for the respective dimensions were incongruent. With this method, subjects must attend to the cued dimension to select the correct response because, on all trials, which response to make to a stimulus depends on the dimension that has been cued. Because the cued dimension varies from trial to trial, subjects cannot be prepared to respond on the basis of a single mapping of stimuli to responses for a particular dimension, as in previous studies of the right-left prevalence effect. At a short cue-target interval, there is insufficient time to prepare fully for the cued dimension in advance of the target stimulus, whereas at a long interval, there should be. Any component of the right-left prevalence effect due to the relative salience or availability of the spatial codes for the two dimensions should be present at short cue-target intervals, yielding a right-left prevalence effect. Because a long cue-target interval allows time to prepare for the cued dimension, responses should be faster than at the short cue-target interval. If this preparation is distinct from the relative salience of dimensions that produces the prevalence effect, as Vu and Proctor $(2001,2002)$ claimed, then no interaction of that effect with cue-target interval should occur. If, instead, the stimulus and response sets are reorganized to prepare for the cued dimension, as Adam et al. (2003) claimed, then right-left prevalence should be reduced at long cue-target intervals. 


\section{EXPERIMENT 1}

In Experiment 1, short and long cue-target intervals were used. This was achieved by using two alternative timelines. For the first, the response-cue interval was constant, which means that the response-stimulus interval (RSI) was longer when the cue-target interval was long than when it was short (cf. Logan \& Bundesen, 2003). Since residual effects from the previous trial should dissipate over time, there is a possibility for stronger carryover effects at the short cue-target interval than at the long one. For the second timeline, the response-cue interval was manipulated to offset the difference in cue-target interval in such a way that the overall RSI was held constant (cf. Meiran, 1996). These RSI conditions were originally conducted as two experiments with slightly different long cue-target intervals (800 and $900 \mathrm{msec}$, respectively). However, we report them together because the pattern of cuing effects for the two RSI methods was similar.

Responding in this task should be based solely on the mapping for the cued dimension (because responding based on the same mapping for the alternative dimension would lead to an error). However, it is possible, though unlikely, that subjects could adopt a strategy of responding on the basis of a single dimension by changing the stimulus-response mapping as a function of the cue. For example, if there is a bias to respond only on the basis of the horizontal dimension, then subjects could respond compatibly when the horizontal dimension is cued and incompatibly on the horizontal dimension when the vertical dimension is cued. To rule out this possibility, we instructed one group to respond to the cued dimension with a compatible mapping and another group to respond to the cued dimension with an incompatible mapping. If subjects are set to respond to the cued dimension with the assigned mapping, a similar right-left prevalence effect should be obtained for both mapping conditions. However, if subjects are biased to respond on the basis of the horizontal dimension alone, the right-left prevalence effect for the compatible mapping condition should reverse to a topbottom prevalence effect for the incompatible mapping condition because incompatibility on the vertical dimension reflects compatibility on the horizontal dimension.

\footnotetext{
Method

Subjects. Sixty-four student volunteers were recruited from Purdue University's introductory psychology courses for credit toward their experimental participation requirement.

Apparatus and Stimuli. The program was written in Micro Experimental Laboratory (2.01) and run on a personal computer. Stimuli were presented on a 14-in. VGA monitor at a distance of $55 \mathrm{~cm}$ from the subject. A double-arrow cue $\left[1.25 \mathrm{~cm}\right.$ long $\left(1.3^{\circ}\right)$ and $0.7 \mathrm{~cm}$ tall $\left(.73^{\circ}\right)$, pointing left/right $(\leftrightarrow)$ or top/bottom $\left.(\uparrow)\right]$ was used to signal the dimension on which responding was to be based. The target stimuli were solid disks $\left(1.5 \mathrm{~cm}, 1.56^{\circ}\right.$ in diameter) arrayed along one of the two major diagonals of an imaginary square (upper left/lower right or upper right/lower left). The diagonal distance between the two alternative target stimuli was $21 \mathrm{~cm}$. Responses were also arrayed along one of two diagonals ( 7 and 3 keys or 9 and 1 keys of a numeric pad of the keyboard). The stimuli were presented along one diagonal, and the responses were made along
}

the alternative diagonal (i.e., upper left/lower right stimuli mapped to upper right/lower left keys, or vice versa). With this arrangement, the stimulus corresponded to the response location along either the horizontal or the vertical dimension, but not both.

Procedure. Half of the subjects were instructed to respond with a compatible stimulus-response mapping along the cued dimension (see Figure 1). If the cue was a left/right double arrow, the left key was to be pressed to the left stimulus and the right key to the right stimulus (note that this mapping is incompatible along the vertical dimension). If the cue was an up/down double arrow, the upper key was to be pressed to the top stimulus and the lower key to the bottom stimulus (this mapping is incompatible along the horizontal dimension). The remaining subjects were instructed to respond with an incompatible mapping along the cued dimension. If the cue was a left/right double arrow, the left key was to be pressed to the right stimulus and the right key to the left stimulus (this mapping is compatible along the vertical dimension). If the cue was an up/down double arrow, the upper key was to be pressed to the bottom stimulus and the lower key to the top stimulus (this mapping is compatible along the horizontal dimension).

The diagonal along which the responses were positioned was counterbalanced between subjects. Subjects performed eight blocks of 80 trials, with the cue-target interval alternating between blocks. The cue-target interval for the first block of trials was also counterbalanced between subjects. Because the stimulus-response relation was compatible along one dimension and incompatible along the alternative dimension, the correct response was dependent on the cue, which was presented at a short or long cue-target interval. For the varied RSI condition, the cue was presented for 100 or $800 \mathrm{msec}$, with a 1-sec response-cue interval. Thus, for this group, the RSI varied between 1,100 and 1,800 msec. For the constant RSI condition, the RSI was held at a constant $2 \mathrm{sec}$. This was accomplished by presenting a blank screen for $1,900 \mathrm{msec}$ followed by the 100 -msec cue or for 1,100 msec followed by a $900-\mathrm{msec}$ cue.

\section{Results}

In all experiments, the first 10 trials in each block were excluded as warm-up, as were trials with reaction time (RT) $<200$ or $>3,000 \mathrm{msec}(<0.67 \%$ of the trials in each experiment). Mean correct RT and percentage error (PE) were submitted to 2 (cue dimension: horizontal or vertical) $\times 2$ (cue-target interval: short or long) $\times 2$ (mapping instructions: compatible or incompatible) $\times 2$ (RSI: varied or constant) ANOVAs (see Table 1 for means). Cue dimension and cue-target interval were within-subjects factors, and mapping instructions and RSI were betweensubjects factors.

Reaction time. Only the main effects of cue dimension $\left[F(1,60)=15.64, M S_{\mathrm{e}}=2,569, p<.001\right]$ and cue-target interval $\left[F(1,60)=41.15, M S_{\mathrm{e}}=6,125, p<.001\right]$ were significant. RT was $25 \mathrm{msec}$ shorter when the cue dimension was horizontal $(M=579 \mathrm{msec})$ than when it was vertical $(M=604 \mathrm{msec})$ and $62 \mathrm{msec}$ shorter when the cue-target interval was long $(M=560 \mathrm{msec})$ than when it was short $(M=622 \mathrm{msec})$. There was no significant main effect of mapping instructions or RSI $(F \mathrm{~s}<1.0)$, nor were there higher level interactions involving any variables, with only the cue dimension $\times$ RSI interaction showing a probability less than $.10\left[F(1,60)=3.53, M S_{\mathrm{e}}=2,569\right.$, $p=.065]$. The trend for this interaction was for the advantage for the horizontal dimension to be larger in the constant RSI condition $(38 \mathrm{msec})$ than in the varied RSI condition (13 msec). 
Table 1

Mean Reaction Time (RT, in Milliseconds) and Percentage Error (PE) in Experiment 1 as a Function of Cue Dimension, Cue-Target Interval, Mapping, and Response-Stimulus Interval (RSI)

\begin{tabular}{|c|c|c|c|c|}
\hline \multirow[b]{3}{*}{ Cue Dimension } & \multicolumn{4}{|c|}{ Cue-Target Interval } \\
\hline & \multicolumn{2}{|c|}{ Short } & \multicolumn{2}{|c|}{ Long } \\
\hline & RT & PE & RT & PE \\
\hline \multicolumn{5}{|c|}{ Compatible Mapping: Varied RSI } \\
\hline Vertical & 629 & 2.72 & 563 & 3.60 \\
\hline Horizontal & 617 & 2.53 & 554 & 1.84 \\
\hline \multicolumn{5}{|c|}{ Compatible Mapping: Constant RSI } \\
\hline Vertical & 631 & 2.62 & 566 & 3.17 \\
\hline Horizontal & 608 & 1.95 & 540 & 2.94 \\
\hline \multicolumn{5}{|c|}{ Incompatible Mapping: Varied RSI } \\
\hline Vertical & 597 & 3.41 & 567 & 4.64 \\
\hline Horizontal & 584 & 2.42 & 549 & 2.24 \\
\hline \multicolumn{5}{|c|}{ Incompatible Mapping: Constant RSI } \\
\hline Vertical & 683 & 3.48 & 594 & 5.76 \\
\hline Horizontal & 631 & 2.91 & 546 & 2.74 \\
\hline
\end{tabular}

Percentage error. The cue dimension main effect was significant $\left[F(1,60)=21.90, M S_{\mathrm{e}}=4.21, p<.001\right]$. Consistent with the RT data, fewer errors were made when the cue dimension was horizontal $(M=2.45 \%)$ than when it was vertical $(M=3.68 \%)$. Cue dimension interacted with cue-target interval $\left[F(1,60)=9.52, M S_{\mathrm{e}}=2.60, p=\right.$ $.003]$. The difference in error rates for the horizontal and vertical dimensions was smaller at the short cue-target interval $(M \mathrm{~s}=2.45 \%$ and $3.06 \% ; p=.062)$ than at the long interval $(M \mathrm{~s}=2.44 \%$ and $4.29 \% ; p<.001)$.

\section{Discussion}

A right-left prevalence effect of $25 \mathrm{msec}$ was evident that did not interact significantly with mapping condition, indicating that subjects were indeed basing their responses on the cued dimension. Responses were $62 \mathrm{msec}$ faster at the long cue-target interval than at the short one, suggesting that subjects were indeed preparing for the cued dimension. Despite this decrease in RT at the long interval, the magnitude of the right-left prevalence effect did not vary as a function of cue-target interval. This pattern of results supports the view that the prevalence effect is determined primarily by the task structure. Processes invoked by instructions to attend to one dimension or the other, either between trial blocks, as in previous studies, or within trial blocks, as in the present experiment, are constrained by the representations generated by the environment. For situations of the type examined in this study, the task structure provides a more salient reference frame for horizontal than for vertical coding $(\mathrm{Vu}$, Proctor, \& Pick, 2000), yielding a right-left prevalence effect.

RSI condition had little effect, with only the interaction with cue dimension approaching significance: The rightleft prevalence effect tended to be larger when RSI was constant than when it varied. More important, RSI did not enter into any interaction involving cue-target interval, suggesting that the precuing effects were due primarily to the time available for preparation and not to changes in residual carryover effects from the previous trial.

Responses were no faster for the groups instructed to respond with a compatible mapping of stimuli to responses on the cued dimension than for those instructed to respond with an incompatible mapping. The lack of compatibility effect likely is due to the fact that, with this cuing procedure, the actual mapping of stimuli to responses varies from trial to trial. Mixing mappings eliminates the benefit for the compatible mapping in two-choice tasks using visuospatial stimulus-response sets (Shaffer, 1965; Vu \& Proctor, 2004). In other words, a compatible mapping is beneficial only when the mapping of stimuli to responses is consistent across trials.

The PE data showed an increase in PE for the vertical dimension relative to the horizontal dimension at the long cue-target interval. This interaction could be interpreted as indicating an increase in the right-left prevalence effect. However, the PE data in Experiment 2 showed the opposite pattern and those for the analogous trials in Experiment 3 showed no significant interaction. Thus, across experiments, there was no consistent pattern to the change in PE for the two dimensions across cue-target interval.

\section{EXPERIMENT 2}

Experiment 1 showed no reduction in the right-left prevalence effect at the long cue-target interval, suggesting that organizational constraints imposed by the task structure cannot be overcome simply by attending to a particular dimension. However, the possibility remained that the duration of the long cue-target interval ( 800 and $900 \mathrm{msec}$ for the varied and constant RSI conditions, respectively) was not sufficient to allow for full preparation of the task set to respond to the cued dimension. For example, Meiran's (2000) Figure 16.2 shows results for task-switching costs in which a large reduction did not occur until a cue-target interval of approximately 1,400 msec, and Reeve and Proctor (1984) found that a cue-target interval longer than $1,500 \mathrm{msec}$ in a four-choice spatial task was needed to produce a benefit for cuing specific pairs of responses in comparison with when all four alternatives remained possible. Experiment 2 was conducted to address this issue, with cue-target intervals of 300 and 3,000 msec.

\section{Method}

Thirty-two new subjects were recruited from the same subject pool as in Experiment 1. Because that experiment showed no significant effect of the RSI manipulation, only the constant RSI was used. As in Experiment 1, mapping was manipulated between subjects. Thus, the only difference in the present experiment from the constant RSI conditions of Experiment 1 was that the RSI was held constant at $4 \mathrm{sec}$. This was accomplished by presenting a blank screen for $3,700 \mathrm{msec}$, followed by the 300-msec cue for the short cue-target interval condition, and a blank screen for $1,000 \mathrm{msec}$ followed by the 3,000-msec cue for the long cue-target interval condition.

\section{Results}

Mean correct RT and PE were submitted to separate 2 (cue dimension: horizontal or vertical) $\times 2$ (cue interval: short or long) $\times 2$ (mapping instructions: compatible 
Table 2

Mean Reaction Time (RT, in Milliseconds) and Percentage Error (PE) in Experiment 2 as a Function of Cue Dimension, Cue-Target Interval, and Mapping

\begin{tabular}{lcccc}
\hline & \multicolumn{4}{c}{ Cue-Target Interval } \\
\cline { 2 - 3 } \cline { 4 - 5 } Cue Dimension & \multicolumn{2}{c}{ Short } & & \multicolumn{2}{c}{ Long } \\
\cline { 2 - 5 } & RT & PE & & PE \\
\hline Vertical & 524 & 2.82 & 565 & 2.46 \\
Horizontal & 529 & 1.42 & 555 & 2.36 \\
& Incompatible Mapping & & \\
Vertical & 527 & 3.05 & 586 & 2.98 \\
Horizontal & 493 & 1.14 & 547 & 1.99 \\
\hline
\end{tabular}

or incompatible) ANOVAs (see Table 2 for means). Cue dimension and cue interval were within-subjects factors, and mapping instruction was a between-subjects factor.

Reaction time. Only the main effects of cue dimension $\left[F(1,30)=5.23, M S_{\mathrm{e}}=2,333, p=.029\right]$ and cue-target interval $\left[F(1,30)=7.88, M S_{\mathrm{e}}=8,304, p=.009\right]$ were significant. RT was $20 \mathrm{msec}$ shorter when the cue dimension was horizontal $(M=531 \mathrm{msec})$ than when it was vertical $(M=551 \mathrm{msec})$ and $45 \mathrm{msec}$ shorter when the cue-target interval was short $(M=518 \mathrm{msec})$ rather than long $(M=$ $563 \mathrm{msec}$ ), which is to be expected since the long interval was $3 \mathrm{sec}$ (see the Discussion section). There was no effect of mapping instructions $(F<1.0)$, nor were there higher level interactions involving any variables, with only the cue dimension $\times$ mapping instructions interaction having a probability less than $.19\left[F(1,30)=3.85, M S_{\mathrm{e}}=2,333\right.$, $p=.059]$. For the compatible mapping, RT was similar for both dimensions; for the incompatible mapping, RT was $36 \mathrm{msec}$ shorter when the cued dimension was horizontal than when it was vertical.

Percentage error. The main effect of cue dimension was significant $\left[F(1,30)=10.81, M S_{\mathrm{e}}=3.57, p=.003\right]$. Consistent with the RT data, fewer errors were made when the cue dimension was horizontal $(M=1.73 \%)$ than when it was vertical $(M=2.83 \%)$. Cue dimension interacted with cue-target interval $\left[F(1,30)=4.33, M S_{\mathrm{e}}=2.25\right.$, $p=.046]$. The difference in error rates for the horizontal and vertical dimensions was smaller at the short cuetarget interval $(M \mathrm{~s}=1.28 \%$ and $2.93 \% ; p<.001)$ than at the long interval $(M \mathrm{~s}=2.17 \%$ and $2.72 \% ; p=.27)$.

\section{Discussion}

RT was longer at the 3,000-msec cue-target interval than at the $300-\mathrm{msec}$ interval. This outcome may initially seem surprising, but it is consistent with other findings obtained when the interval between an uninformative warning signal and the target stimulus is varied in two-choice spatial tasks. Posner, Klein, Summers, and Buggie (1973) had subjects perform using a compatible or incompatible mapping, with blocked foreperiods of 0, 50, 100, 200, 400, or $800 \mathrm{msec}$. RT was a reversed J-shaped function of foreperiod, with the longest RT for both mappings evident at the two shortest foreperiods and the shortest RT between the 100- and 400-msec foreperiods. Thus, the 300-msec cue-target interval in the present study was in the range for optimal alertness, but the 3,000-msec interval was outside of this range. It is worth noting, though, that RT at the 3,000-msec cue-target interval was still $59 \mathrm{msec}$ shorter than that obtained at the 100-msec cue-target interval in Experiment 1, suggesting that subjects were prepared to respond on the basis of the cued dimension.

A right-left prevalence effect was clearly evident only with the incompatible mapping. We do not know why the compatible mapping showed no clear prevalence effect, but the tendency for the effect to be larger with the incompatible mapping was in agreement with the results of Experiment 1 . Of most importance, the right-left prevalence effect was equally apparent at the 300- and 3,000-msec cue-target intervals, indicating that the effect was not restricted solely to short intervals.

\section{EXPERIMENT 3}

As we noted in the introduction, Meiran (1996) used a method similar to that of the present Experiments 1 and 2 to study task switching. With his method, a target stimulus is presented in one of four quadrants of a display screen, and responses are made with index fingers placed on the positive ( 1 and 9 keys) or negative ( 3 and 7 keys) diagonal of a numeric keypad, as in the present study. The precue consists of two outward pointing arrows located at the ends of the horizontal or vertical dividing line to indicate whether the task is to respond with a left-right mapping or a top-bottom mapping, respectively. With this method, the relevant $\mathrm{S}-\mathrm{R}$ mapping is compatible for both dimensions. Consequently, the same response is made for both mappings when the stimulus diagonal corresponds to the response diagonal (rule-congruent trials) but not when the stimulus diagonal corresponds to the alternative diagonal (rule-incongruent trials).

Although his main interest was in task switching, Meiran (1996) presented the results of his Experiment 4 as a function of task (top-bottom, right-left). Task was not significant on its own or in combination with any other variable. However, RT was $10 \mathrm{msec}$ shorter for the right-left task than the top-bottom task, and this difference was largest for rule-incongruent trials on which there was a task switch (34 msec at the short cue-target interval and $19 \mathrm{msec}$ at the long cue-target interval). Meiran et al. (2000) showed significant effects of this type in their Experiment 2, in which the response-cue interval was blocked at different values and the cue-target interval varied randomly among $116,316,516$, and 2,016 msec. RT was $24 \mathrm{msec}$ shorter for the right-left task than for the top-bottom task, and the advantage for the former task was larger at the short cue-target intervals than at the longer ones (RT differences of $35,33,12$, and $15 \mathrm{msec}$, respectively). Meiran et al. replicated the right-left prevalence effect in their Experiment 3, but reported that there was no significant interaction with cue-target interval.

Across the experiments of Meiran (1996) and Meiran et al. (2000), it is clear that a right-left prevalence effect was present. However, unlike our results, this effect seemed to 
decrease at longer cue-target intervals, as predicted by Adam et al. (2003), although the interaction was significant in only one of the three experiments for which the relevant data were reported. If this apparent reduction in the right-left prevalence effect at longer cue-target intervals is reliable, then one possible reason why this pattern was not evident in the present Experiments 1 and 2 may be that these experiments did not include rule-congruent trials. Another way in which our experiments differed from those of Meiran and colleagues is that we used blocked cue-target intervals instead of randomizing the intervals with a trial block.

Because the present study showed no decrease of the right-left prevalence effect at long cue-target intervals (i.e., with preparation), we wanted to rule out the possibility that this null effect was due to specifics of our methods. Therefore, in Experiment 3 we evaluated whether inclusion of rule-congruent trials or blocked versus randomized presentation of cue-target intervals would influence the right-left prevalence effect. A stimulus was presented in one of four possible corners of an imaginary square. Responses were arrayed along either a positive or a negative diagonal of the numeric keypad, counterbalanced between subjects. With this arrangement, the mapping of stimuli to responses was compatible (or incompatible) along one dimension when the stimuli occurred along the opposite diagonal from that of the responses (rule-incongruent trials), as in Experiments 1 and 2, or along both dimensions when the stimuli occurred along the same diagonal as that for the responses (rule-congruent) trials. All subjects were instructed to respond to the location of the stimuli with a compatible mapping on the cued dimension.

\section{Method}

Thirty-two new volunteers were recruited from the same subject pool as in Experiments 1 and 2. The short and long cue-target intervals of 100 and $900 \mathrm{msec}$ were used, as in Experiment 1, along with a constant RSI of $2 \mathrm{sec}$. Each subject received a total of 640 trials. In the randomized cue-target interval condition, subjects received four blocks of 160 trials. The first 20 trials of each block were excluded as practice. For the blocked cue-target conditions, subjects received eight blocks of 80 trials, with the short and long cue-target intervals alternating between blocks of trials. The first 10 trials of each block were excluded as practice.

\section{Results}

Mean correct RT and PE were submitted to separate 2 (cue dimension: horizontal or vertical) $\times 2$ (cue-target interval: short or long) $\times 2$ (rule congruency: congruent or incongruent trials) $\times 2$ (block type: blocked or randomized intervals) ANOVAs (see Table 3 for means). Cue dimension, cue-target interval, and rule congruency were within-subjects factors, and block type was a betweensubjects factor.

Reaction time. The main effects of cue dimension $\left[F(1,30)=11.55, M S_{\mathrm{e}}=999, p=.002\right]$, cue-target interval $\left[F(1,30)=16.70, M S_{\mathrm{e}}=8,110, p<.001\right]$, and rule congruency $\left[F(1,30)=106.54, M S_{\mathrm{e}}=5,740, p<.001\right]$ were significant. Responses were $13 \mathrm{msec}$ faster when the cue dimension was horizontal $(M=532 \mathrm{msec})$ than
Table 3

Mean Reaction Time (RT, in Milliseconds) and Percentage Error (PE) in Experiment 3 as a Function of Cue Dimension, Congruency, Cue-Target Interval, and Block Type

\begin{tabular}{lcccc}
\hline & \multicolumn{4}{c}{ Cue-Target Interval } \\
\cline { 2 - 5 } Cue Dimension & RT & PE & RT & PE \\
\cline { 2 - 5 } \cline { 3 - 4 } Vertical & Blocked Presentation: Congruent Trials & \\
Horizontal & 511 & 0.26 & 471 & 0.01 \\
& 498 & 0.46 & 468 & 0.17 \\
Vertical & Blocked Presentation: Incongruent Trials & \\
Horizontal & 605 & 4.26 & 574 & 5.09 \\
& 598 & 2.25 & 552 & 3.57 \\
Vertical & Random Presentation: Congruent Trials & \\
Horizontal & 521 & 0.35 & 464 & 0.01 \\
& 528 & 0.01 & 459 & 0.00 \\
Vertical & Random Presentation: Incongruent Trials & \\
Horizontal & 627 & 5.00 & 593 & 3.97 \\
\hline
\end{tabular}

when it was vertical $(M=545 \mathrm{msec})$ and $46 \mathrm{msec}$ faster when the cue-target interval was long $(M=516 \mathrm{msec})$ than when it was short $(M=562 \mathrm{msec})$. Responses were also faster for rule-congruent trials $(M=490 \mathrm{msec})$ than rule-incongruent trials $(M=588 \mathrm{msec})$. There was no significant main effect of block type $(F<1.0)$.

The only significant interaction was that of rule congruency and cue dimension $\left[F(1,30)=6.16, M S_{\mathrm{e}}=1,159\right.$, $p=.019]$. There was no right-left prevalence effect on rule-congruent trials (mean difference, $M D=3 \mathrm{msec}$ ) $[F(1,31)=1.03, p>.05]$, but a right-left prevalence effect was evident on rule-incongruent trials $(M D=$ $24 \mathrm{msec})[F(1,31)=9.43, p=.004]$. The only other term in the ANOVA with a probability less than .10 was the cue dimension $\times$ cue-target interval interaction $[F(1,30)=$ $\left.3.15, M S_{\mathrm{e}}=521, p=.086\right]$. The trend was for the rightleft prevalence effect to be smaller at the short cue-target interval ( $8 \mathrm{msec})$ than at the long one $(19 \mathrm{msec})$.

Percentage error. The main effects of cue dimension $\left[F(1,30)=27.13, M S_{\mathrm{e}}=3.52, p<.001\right]$ and rule congruency $\left[F(1,30)=4.61, M S_{\mathrm{e}}=13.56, p<.001\right]$ were significant. Consistent with the RT data, fewer errors were made when the cue dimension was horizontal $(M=1.17 \%)$ than when it was vertical $(M=2.39 \%)$, and when trials were rule congruent $(M=0.19 \%)$ than when they were rule incongruent $(M=3.37 \%)$. The only other significant effect was the two-way interaction of rule congruency $\times$ cue dimension $\left[F(1,30)=27.94, M S_{\mathrm{e}}=3.34, p<.001\right]$. There was no evidence of a right-left prevalence effect on rulecongruent trials $(M D=0.01 \%)(F<1.0)$, but an effect of $2.43 \%$ was evident on rule-incongruent trials $[F(1,31)=$ $27.62, p<.001]$. Unlike in Experiments 1 and 2, the ruleincongruent trials did not show a significant interaction of cue dimension $\times$ cue-target interval $(F<1.0)$.

\section{Discussion}

As in Experiments 1 and 2, a right-left prevalence effect was obtained that did not vary significantly as a func- 
tion of cue-target interval, though it did tend to be larger at the long than at the short interval. Furthermore, similar effects were obtained when the long and short cue-target intervals were randomly intermixed and when they were blocked. Rule congruency influenced the right-left prevalence effect significantly, with the prevalence effect being evident only for rule-incongruent trials. The magnitude of the right-left prevalence effect for those trials $(24 \mathrm{msec})$ was comparable to that of the effects obtained in Experiments $1(25 \mathrm{msec})$ and $2(20 \mathrm{msec})$, which contained only rule-incongruent trials. Thus, the presence of rulecongruent trials did not alter the magnitude of the prevalence effect for rule-incongruent trials.

We clearly did not find a decrease of the right-left prevalence effect at the long cue-target interval. Thus, the absence of such a tendency in Experiments 1 and 2 is not due to blocking the intervals or to inclusion of only ruleincongruent trials. Given that this tendency was significant in only one of Meiran's (1996) and Meiran et al.'s (2000) three experiments mentioned earlier, it does not seem to be a general property of results in the rule-congruent/ -incongruent task.

The method of Experiment 3 was similar in several respects to that used by Meiran (1996; Meiran et al., 2000) in his studies of task-switching, where he found that the cost for switching between the horizontal and vertical dimensions, or tasks, was reduced at long cue-target intervals. Consequently, we conducted an analysis of the RT data with task repetition (the cued dimension repeated or switched) as an additional factor. Although RT was shorter when the task was repeated $(M=522 \mathrm{msec})$ than when it was switched $(M=555 \mathrm{msec})\left[F(1,30)=54.31, M S_{\mathrm{e}}=\right.$ $1,312, p<.001]$, task repetition did not interact with cue-target interval alone or in combination with any other variable $\left(F_{\mathrm{S}}<1.0\right)$. We performed an additional analysis on subjects from all three experiments (see Table 4), with experiment, task repetition, cue dimension, and cuetarget interval as variables. This analysis also showed no interaction of task repetition $\times$ cue-target interval $\left(F_{\mathrm{S}}<\right.$ $1.0)$, indicating that the absence of an effect of cue-target interval on the task-switching cost generalized across our other experiments in which all trials were incongruent.

Table 4

Mean Reaction Time (in Milliseconds) in Experiments 1, 2, and 3 as a Function of Task Repetition and Cue-Target Interval

\begin{tabular}{lrr}
\hline & \multicolumn{2}{c}{ Cue-Target Interval } \\
\cline { 2 - 3 } Task Repetition & Short & Long \\
\hline & Experiment 1 & \\
Repetition & 585 & 521 \\
Switch & 659 & 595 \\
& Experiment 2 & \\
Repetition & 492 & 535 \\
Switch & 544 & 591 \\
& Experiment 3 & \\
Repetition & 545 & 499 \\
Switch & 579 & 531 \\
\hline
\end{tabular}

The absence of a "switch preparation effect" in which the switch trials benefit from a longer cue-target interval more than do the nonswitch trials is not without precedent (Altmann, 2004; Koch, 2001). However, the interaction of task repetition $\times$ cue-target interval has proven to be replicable (e.g., Koch et al., 2003) with close variants of the specific cue stimuli and display used by Meiran (1996; Meiran et al., 2000). The present experiments differed from Meiran's in that the screen was clear instead of being divided into quadrants by gridlines, the cue stimulus was a centered double arrow instead of arrowheads presented outside the center axes of the grid, and the target stimulus was not presented in a marked quadrant. One of these differences may be critical in determining whether a switch preparation effect is obtained.

\section{GENERAL DISCUSSION}

The right-left prevalence effect has previously been studied under conditions in which the mapping of stimuli to responses and the instructed dimension are held constant in a trial block. With that procedure, subjects are prepared to respond as they have been instructed on all trials. Thus, it is not possible to examine whether the prevalence effect varies as a function of state of preparation. In the present study, the cued dimension varied from trial to trial. By varying the interval between the onsets of the cue that signaled the relevant dimension and the target stimulus, it was possible to vary the opportunity to prepare for responding on the basis of the cued dimension. If attentional orientation to one dimension or the other is separate from the environmentally constrained codes that are responsible for the right-left prevalence effect, then the benefits of cuing the horizontal or vertical dimension in advance should be independent of the right-left prevalence effect.

In all experiments, RT varied as a function of cuetarget interval, but the right-left prevalence effect occurred independently of the interval length. This outcome implies that attention can be directed to either dimension, as Hommel (1996) showed with his instruction manipulation, but this attentional allocation is not the source of the right-left prevalence effect. Rather, the outcome is in accord with Vu and Proctor's $(2001,2002)$ proposal that the prevalence effect is mainly a consequence of the relative salience of horizontal and vertical codes provided by the task structure. The right-left prevalence effect for ruleincongruent conditions of previous experiments that used a display and response apparatus similar to those in the present study was approximately $20 \mathrm{msec}$ (Vu et al., 2000, Experiment 2, constant practice condition; Vu \& Proctor, 2002, Experiment 2, equivalent display condition), a size comparable to the $23-\mathrm{msec}$ effect obtained in the present three experiments. This finding agrees with the view that preparation has little influence on the right-left prevalence effect, and it is contrary to the implication of Adam et al.'s (2003) grouping model that slow, effortful preparation can overcome the stimulus and response subgroups that are produced more automatically. 
Meiran (2005) recently reported an experiment in which he investigated task switching using (1) rule-congruent and rule-incongruent stimuli and (2) compatible and incompatible mappings of the relevant stimulus dimension to responses. Although Meiran (2005) did not examine the right-left prevalence effect, his data are generally consistent with those from our experiments. There was little difference between the horizontal and vertical tasks for rule-congruent trials, but responses were $65 \mathrm{msec}$ faster on the horizontal task than on the vertical task for rule-incongruent trials. This pattern indicates a right-left prevalence effect only for rule-incongruent trials, as in the present Experiment 3. Furthermore, mapping condition did not enter into a twoway interaction with task, indicating that the right-left prevalence effect was equally evident for the two mappings. This outcome is similar to those of the present Experiments 1 and 2, in showing that the incompatible mapping condition does not reverse the right-left prevalence effect.

If the right-left prevalence effect in tasks structured like those of the present experiments is solely a consequence of the physical layout of stimuli and responses, then a similar prevalence effect should be evident in tasks using Simontype stimuli, for which stimulus location is irrelevant and color (or another nonspatial attribute) is relevant. Rubichi, Nicoletti, and Umiltà (2005) recently reported finding a right-left prevalence effect using Simon stimuli. In their Experiment 2, the stimuli were colored squares presented in one of the four corners of an imaginary square, and responses were made by a contralateral hand and foot arrayed along either the positive or the negative diagonal. With this stimulus and response arrangement, they found a significant Simon effect for the horizontal dimension but not the vertical dimension, indicating a right-left prevalence effect. Rubichi et al. (2005) treated this difference in Simon effects as a prevalence effect, because they considered the contralateral hand and foot to be equally salient on both dimensions. However, we have presented evidence that contralateral effectors tend to be coded as left and right, whereas ipsilateral effectors are coded as top and bottom (Vu \& Proctor, 2001).

Proctor, Vu, and Nicoletti (2003) showed that an advantage for the horizontal or vertical Simon effect can be obtained by altering the relative salience of the stimulusresponse sets to favor one dimension or the other. Moreover, the benefit for horizontal correspondence with horizontally salient sets was not any larger than that for vertical correspondence with vertically salient sets. Hence, Proctor et al. concluded that, although the relative salience of the two dimensions provided by the physical environment can alter the magnitude of the horizontal and vertical Simon effects, there is no inherent benefit for horizontal codes over vertical codes in the Simon task, as there is when stimulus location is relevant. Furthermore, Proctor et al. found that when stimulus locations were equally spaced, with responses made on a numeric keypad, as in the present study, no right-left prevalence effect was evident (see also Vu, Pellicano, \& Proctor, 2005). That Simon tasks show no right-left prevalence effect under conditions that yield an effect when stimulus location is relevant implies that the prevalence effect is not just a consequence of the physical layout of stimuli and responses but also of the requirement to respond to location as the relevant stimulus attribute.

In conclusion, the right-left prevalence effect is obtained when the dimension to which a compatible or incompatible mapping should be applied is cued on a trial-to-trial basis. This effect occurs regardless of whether the preparation time is short or long, and it seems to reflect the relative ease with which the stimulus codes can be intentionally translated into response codes. That the right-left prevalence effect is evident only on rule-incongruent trials suggests that the advantage for horizontal location coding occurs primarily when the horizontal and vertical codes do not signal the same response.

\section{REFERENCES}

Adam, J. J., Hommel, B., \& Umiltà, C. (2003). Preparing for perception and action (I): The role of grouping in the response-cuing paradigm. Cognitive Psychology, 46, 302-358.

Altmann, E. M. (2004). The preparation effect in task switching: Carryover of SOA. Memory \& Cognition, 32, 153-163.

HommeL, B. (1996). No prevalence of right-left over top-bottom spatial codes. Perception \& Psychophysics, 58, 102-110.

$\mathrm{KoCH}$, I. (2001). Automatic and intentional activation of task sets. Journal of Experimental Psychology: Learning, Memory, \& Cognition, 27, 1474-1486.

Koch, I., Ruge, H., Brass, M., Rubin, O., Meiran, N., \& Prinz, W. (2003). Equivalence of cognitive processes in brain imaging and behavioral studies: Evidence from task switching. Neurolmage, 20, 572-577.

Logan, G. D., \& Bundesen, C. (2003). Clever homunculus: Is there an endogenous act of control in the explicit task-cuing procedure? Journal of Experimental Psychology: Human Perception \& Performance, 29, 575-599.

MeIran, N. (1996). Reconfiguration of processing mode prior to task performance. Journal of Experimental Psychology: Learning, Memory, \& Cognition, 22, 1423-1442.

Meiran, N. (2000). Reconfiguration of stimulus task sets and response task sets during task switching. In S. Monsell \& J. Driver (Eds.), Control of cognitive processes: Attention and performance XVIII (pp. 377-399). Cambridge, MA: MIT Press.

Meiran, N. (2005). Task rule-congruency and Simon-like effects in switching between spatial tasks. Quarterly Journal of Experimental Psychology, 58A, 1023-1041.

Meiran, N., Chorev, Z., \& Sapir, A. (2000). Component processes in task switching. Cognitive Psychology, 41, 211-253.

Nicoletti, R., \& Umiltà, C. (1984). Right-left prevalence in spatial compatibility. Perception \& Psychophysics, 35, 333-343.

Nicoletti, R., \& Umiltà, C. (1985). Responding with hand and foot: The right/left prevalence in spatial compatibility is still present. Perception \& Psychophysics, 38, 211-216.

Nicoletti, R., Umiltà, C., Tressoldi, E. P., \& Marzi, C. A. (1988). Why are left-right spatial codes easier to form than above-below ones? Perception \& Psychophysics, 43, 287-292.

Posner, M. I., Klein, R., Summers, J., \& Buggie, S. (1973). On the selection of signals. Memory \& Cognition, 1, 2-12.

Proctor, R. W., Vu, K.-P. L., \& Nicoletti, R. (2003). Does right-left prevalence occur for the Simon effect? Perception \& Psychophysics, 65, 1318-1329.

Reeve, T. G., \& Proctor, R. W. (1984). On the advance preparation of discrete finger responses. Journal of Experimental Psychology: Human Perception \& Performance, 10, 541-553.

Rubichi, S., Nicoletti, R., Pelosi, A., \& Umiltà, C. (2004). Right- 
left prevalence effect with horizontal and vertical effectors. Perception \& Psychophysics, 66, 255-263.

Rubichi, S., Nicoletti, R., \& Umiltà, C. (2005). Right-left prevalence with task-irrelevant spatial codes. Psychological Research, 69, 167-178.

Shaffer, L. H. (1965). Choice reaction with variable S-R mapping. Journal of Experimental Psychology, 70, 284-288.

Umiltà, C., \& Nicoletti, R. (1990). Spatial stimulus-response compatibility. In R. W. Proctor \& T. G. Reeve (Eds.), Stimulus-response compatibility: An integrated perspective (pp. 89-116). Amsterdam: Elsevier, North-Holland.

Vu, K.-P. L., Pellicano, A., \& Proctor, R. W. (2005). No overall right-left prevalence for horizontal and vertical Simon effects. Perception \& Psychophysics, 67, 929-938.

Vu, K.-P. L., \& Proctor, R. W. (2001). Determinants of the right-left and top-bottom prevalence for two-dimensional spatial compatibility.
Journal of Experimental Psychology: Human Perception \& Performance, 27, 813-828.

Vu, K.-P. L., \& Proctor, R. W. (2002). The prevalence effect in twodimensional stimulus-response compatibility is a function of the relative salience of dimensions. Perception \& Psychophysics, 64, 815-828.

Vu, K.-P. L., \& Proctor, R. W. (2004). Mixing compatible and incompatible mappings: Elimination, reduction, and enhancement of spatial compatibility effects. Quarterly Journal of Experimental Psychology, 57A, 539-556.

Vu, K.-P. L., Proctor, R. W., \& Pick, D. F. (2000). Vertical versus horizontal compatibility: Right-left prevalence with bimanual responses. Psychological Research, 64, 25-40.

(Manuscript received July 8, 2004; revision accepted for publication May 8,2005 .) 